\title{
Achieving Perfect Hand Washing: an Audit Cycle with Surgical Internees
}

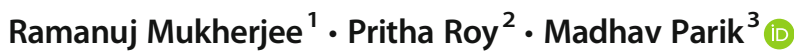 \\ Received: 11 May 2020 / Accepted: 29 September 2020 / Published online: 6 October 2020 \\ (C) Association of Surgeons of India 2020
}

\begin{abstract}
The aim of this study is to achieve $100 \%$ compliance in surgical hand antisepsis along with identification of areas of worst compliance and efficacies of various interventions best suited to deal with them. This audit was performed over 6 days in a tertiary care hospital in Calcutta, India, with 42 surgical internees. Compliance to ideal hand washing technique was recorded after each attempt with the first attempt as baseline. Video demonstration, personal demonstration by a consultant, and individual instruction were used as subsequent interventions to achieve $100 \%$ compliance. The baseline level of compliance was found to be $33.59 \%$. A total of 6 attempts was required to achieve $100 \%$ compliance, with the increase in compliance being statistically significant $(p=0.0294)$. Personal instruction was found to be the most effective intervention. Hand washing technique was the criterion that needed the most number of attempts $(n=6)$ to rectify. This study found video-based instruction and individual guidance effective teaching tools for surgical hand disinfection and gave novel data regarding the reasons responsible for poor compliance to proper hand washing in a general surgical setting. This study demonstrated the efficiency of audit cycles in the improvement of surgical hand washing and can be the preferred mode of intervention in future studies aimed at achieving ideal hand antisepsis.
\end{abstract}

Keywords Asepsis $\cdot$ Hand washing $\cdot$ Clinical audit $\cdot$ Hand hygiene

\section{Introduction}

In the twenty-first century, hand washing has become an essential practice in the field of healthcare, even though the relationship between hand washing and spread of infections was established about two centuries ago [1]. It is well established in current scientific literature that cross infection of patients from the hands of the care

Madhav Parik

madhavparik@gmail.com

Ramanuj Mukherjee

docramu77@gmail.com

Pritha Roy

pritharoy012@gmail.com

1 Department of General Surgery, RG Kar Medical College and Hospital Kolkata, 136 Sarat Bose Road, Calcutta 700029, India

2 Department of Radiotherapy, RG Kar Medical College and Hospital Kolkata, AA-85 Block, Saltlake, Calcutta 700064, India

3 Department of Psychiatry, RG Kar Medical College and Hospital Kolkata, Block C-111, Bangur Avenue, Calcutta 700055, India giver results in healthcare-associated infection (HCAI) $[2,3]$. HCAI is a global problem as it leads to increased morbidity and mortality amongst patients and development of resistance amongst microorganisms due to the rampant use of antimicrobials and is a huge economic burden for patients as well as healthcare systems. HCAI is specially challenging to deal with in developing countries due to limited resources and the lack of reliable investigations, monitoring and standardization of medical records. This along with factors like overcrowding, understaffed healthcare systems and the lack of nationwide surveillance leads to the several-fold higher incidence of HCAIs in developing countries compared with developed countries [4]. Thus in India, education regarding proper hand hygiene in all tiers of healthcare delivery becomes all the more essential. This bears all the more significance with the emergence and global spread of COVID-19, declared a pandemic by the WHO on March 11, 2020. Proper hand washing can help protect healthcare providers from contracting the virus themselves, in turn preventing them from transmitting it further to their patients [5]. 
To reduce the incidence of HCAI, the WHO recommends five situations where hand washing is deemed necessary: (1) before patient contact; (2) before an aseptic task; (3) after body fluid exposure risk; (4) after patient contact; and (5) after contact with patient surroundings [6]. Surgeons all over the world routinely carry out surgical hand antisepsis before undertaking invasive procedures to destroy transient microorganisms and inhibit the growth of resident microorganisms. The World Health Organization (WHO)-recommended guidelines for surgical scrubbing are thus a useful tool for achieving optimum hand hygiene and are followed in our institution. Even though surgical hand scrubbing is practised worldwide, noncompliance has been noted in its various aspects by different researchers $[7,8]$. Thus, an audit cycle was conducted in the general surgery department of our institution with surgical interns to achieve a $100 \%$ compliance rate in surgical hand washing.

\section{Methodology}

This audit cycle was performed in a general surgical unit of a tertiary medical care centre in India, with 42 surgical internees over a period of 6 days from June 1, 2020, to July 1, 2020. On the first day, all participants were asked to wash their hands after entering the operation theatre. This attempt (Attempt 1) was observed, and a video tutorial was then displayed to all candidates not following ideal hand washing protocol [10]. On the following day, the same procedure was repeated, and results were recorded (Attempt 2). For subjects still unable to follow the ideal method, a demonstration of proper technique was done by a consultant surgeon followed by Attempt 3 the next day. Subjects still failing to follow proper procedure were then given personal demonstrations, and individual mistakes were addressed by the consultant. This intervention was done every day till the entire group showed $100 \%$ efficacy on the sixth day (Attempt 6). During each attempt, the participants were observed by three consultants from a single surgical unit, who graded them based on their compliance to the checklist in Table 1. The same protocols were followed by all units of the hospital. Flow chart of Attempts 1-6, the interventions at each step and the success rates have been depicted in Fig. 1.

A total of 42 undergraduate general surgical internees participated in the audit cycle conducted over 6 days with an end goal of $100 \%$ efficacy in hand washing according to the hand washing checklist shown in Table 1 [9]. In Attempt 1 on the first day, 14 and 6 participants were found to comply with only points 1 and 4 of the checklist, respectively, and all of the 42 participants were compliant with the remaining 12 points. Every attempt by each of the participants in each individual checklist criterion was counted as a single unit, and the success rate of each attempt was determined based on the percentage of correct attempts with respect to the total number of attempts. Success units were calculated by adding the total number of new criteria the participants fulfilled after each intervention, and the success rates of the attempts were determined. Once a participant correctly performed one step of the 14 step checklist, he/she was considered to have mastered that aspect of hand washing and was not evaluated on that step in successive attempts. Once all the participants eligible for a particular criterion on the checklist performed it correctly, that criterion was called a successful criterion and was not
Table 1 Checklist to follow for proper hand preparation, washing, and drying [9]

\begin{tabular}{l}
\hline Hand preparation \\
\hline 1. Hands and wrists are free from watches and jewellery (nonstoned wedding rings are acceptable) \\
2. Nails are short and without nail extensions and varnish \\
3. Sleeves are short or rolled up during hand washing \\
4. Cuts are covered with a waterproof dressing \\
Hand washing technique \\
5. Hands are wet under continuously running water \\
6. Warm water is used to wash hands \\
7. Dispensed liquid soap is used \\
8. Liquid soap is applied to wet hands \\
9. Hands are rubbed to create a lather \\
10. A copy of the 10 steps to effective hand hygiene wall chart is prominently displayed at each \\
wash basin \\
11. The lather is rubbed over all surfaces of the hands for 10-15 s, including the thumbs, between \\
the fingers, fingertips and the wrist \\
12. Hand are rinsed thoroughly under running water \\
Drying of hands \\
13. Taps are turned off using wrist/elbow levers or using a clean paper towel \\
14. Hands are dried using paper towels
\end{tabular}


monitored for data collection in the successive attempts (bold marking in Table 2).

\section{Results}

A total of 42 undergraduate general surgical internees were monitored for their hand washing skills, and necessary interventions were carried out for rectification of mistakes in different aspects of hand washing. As the participants improved their hand washing technique, the number of total units in each attempt decreased from 524 in Attempt 1 to 4 in Attempt 6, as shown in Table 2. In Attempt 1 (baseline data), the success rate was $33.59 \%$. After the video demonstration of hand washing, the success rate increased to $41.38 \%$, and after a real life demonstration by the consultant, it increased to $65.69 \%$. Thereafter, three rounds of individual guidance by the consultant were required to increase the success rates from $71.43 \%$ in attempt 4 to $80 \%$ in Attempt 5 and finally $100 \%$ in Attempt 6. The increase in success rates or the improvement of hand washing across the six attempts was found to be statistically significant ( $p=0.0294)$. Maximum number of five successful criteria was found in Attempt 4 after Intervention 3 and thus
Attempt 4 was statistically the most significant attempt; in other words, Intervention 3 which is personal guidance by the consultant was statistically the most significant intervention in improving hand washing practices. Attempt 4 was followed by Attempt 3 with 3 successful criteria, Attempts 1 and 5 ( 2 successful criteria each) and finally by Attempts 2 and 6 ( 1 successful criterion each). Criteria with $>70 \%$ success rate in any attempt are mentioned in italics in Table 2.

According to the individual criterion in the hand washing checklist (Table 1), best adherence was noted in criteria 7 (dispensed liquid soap used) and 10 (a copy of the 10 steps to effective hand hygiene wall chart is prominently displayed at each wash basin) with $100 \%$ success rates for each of the criteria in Attempt 1. In Attempt 2, criterion 8 (liquid soap applied to wet hands) had a success rate of $100 \%$, while in Attempt 3, criteria 1 (hands and wrists are free from watches and jewellery), 3 (sleeves are short or rolled up during hand washing) and 9 (hands are rubbed to create a lather) achieved $100 \%$ success rates. In Attempt 3, maximum number of criteria were seen to achieve a success rate of $>70 \%$ - criteria 4 (cuts are covered with a waterproof dressing), 12(hands are rinsed thoroughly under running water), 13(taps are turned off using wrist/elbow levers or using a clean paper towel) and
Fig. 1 Flowchart displaying the study methodology, with the attempts and interventions made at each step

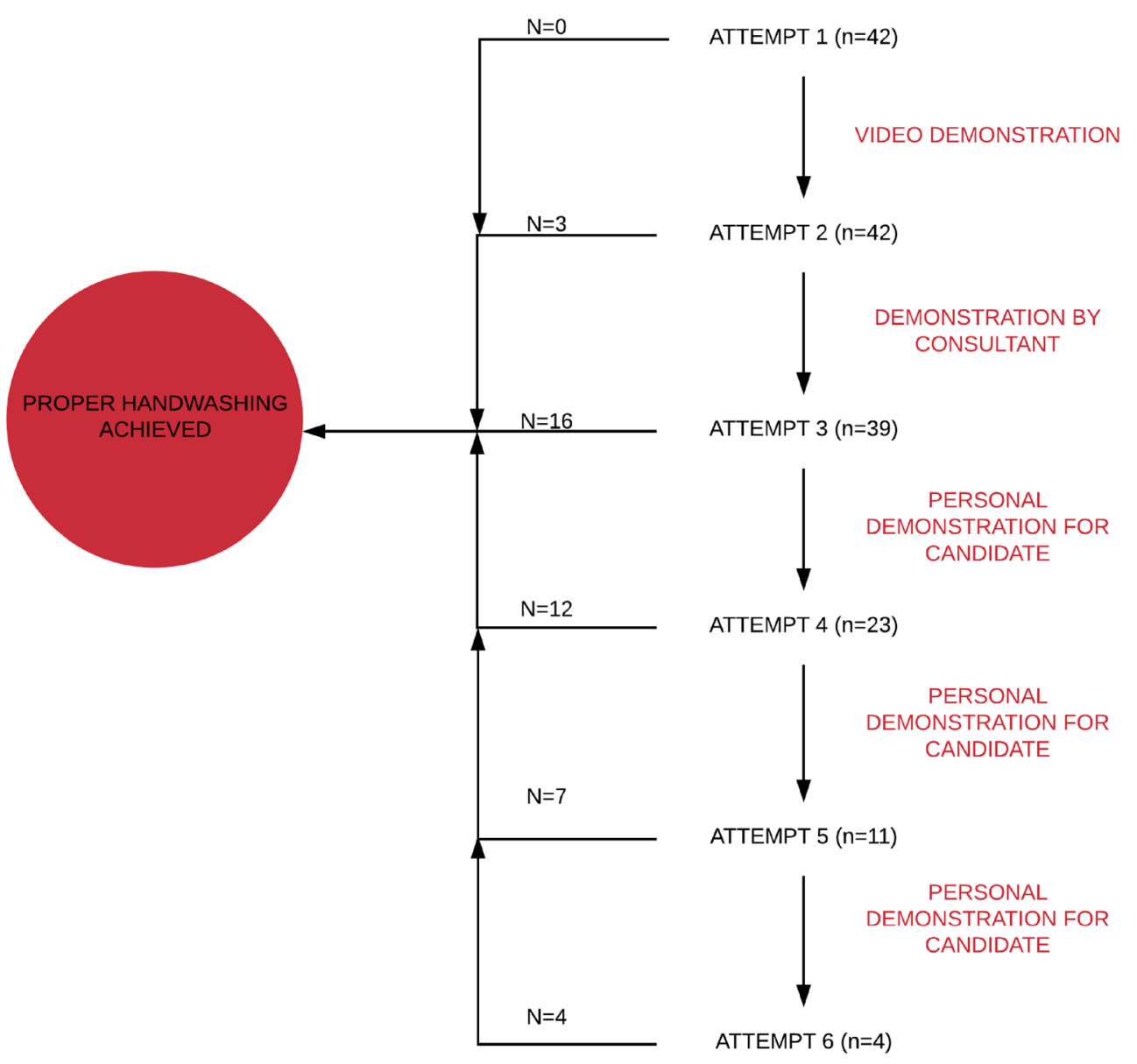


Table 2 Success rates in each attempt according to individual requirements on the hand washing checklist

\begin{tabular}{|c|c|c|c|c|c|c|c|}
\hline \multicolumn{2}{|c|}{ Hand washing checklist } & \multirow{2}{*}{$\begin{array}{l}\text { Attempt } \\
1 \\
28.57\end{array}$} & \multirow{2}{*}{$\begin{array}{l}\text { Attempt } \\
2 \\
90.00\end{array}$} & \multirow{2}{*}{$\begin{array}{l}\text { Attempt } \\
3 \\
\mathbf{1 0 0}\end{array}$} & \multirow{2}{*}{$\begin{array}{l}\text { Attempt } \\
4 \\
-\end{array}$} & \multirow{2}{*}{$\begin{array}{l}\text { Attempt } \\
5\end{array}$} & \multirow{2}{*}{$\begin{array}{l}\text { Attempt } \\
6 \\
-\end{array}$} \\
\hline Hand preparation & Hands and wrists are free from watches and jewellery & & & & & & \\
\hline & Nails are short and without nail extensions and varnish & 30.95 & 68.97 & 66.67 & 100 & - & - \\
\hline & Sleeves are short or rolled up during hand washing & 33.33 & 89.29 & 100 & - & - & - \\
\hline & Cuts are covered with a waterproof dressing & 16.67 & 0.00 & 80.00 & 100 & - & - \\
\hline \multirow{8}{*}{$\begin{array}{l}\text { Hand washing } \\
\text { technique }\end{array}$} & Hands are wet under continuously running water & 0.00 & 14.29 & 61.11 & 71.43 & 100 & - \\
\hline & Warm Water is used to wash hands & 0.00 & 14.29 & 52.78 & 70.59 & 100 & - \\
\hline & Dispensed liquid soap is used & 100 & - & - & - & - & - \\
\hline & Liquid soap is applied to wet hands & 73.81 & 100 & - & - & - & - \\
\hline & Hands are rubbed to create a lather & 21.43 & 69.70 & 100 & - & - & - \\
\hline & $\begin{array}{l}\text { A copy of the } 10 \text { steps to effective hand hygiene wall } \\
\text { chart is prominently displayed at each wash basin }\end{array}$ & 100 & - & - & - & - & - \\
\hline & $\begin{array}{l}\text { The lather is rubbed over all surfaces of the hands for } \\
10-15 \mathrm{~s} \text {, including the thumbs, between the fingers, } \\
\text { fingertips and the wrist }\end{array}$ & 0.00 & 7.14 & 41.03 & 52.17 & 63.64 & 100 \\
\hline & Hand are rinsed thoroughly under running water & 11.90 & 24.32 & 75.00 & 100 & - & - \\
\hline \multirow[t]{2}{*}{ Drying of hands } & $\begin{array}{l}\text { Taps are turned off using wrist/elbow levers or using } \\
\text { a clean paper towel }\end{array}$ & 16.67 & 74.29 & 77.78 & 100 & - & - \\
\hline & Hands are dried using paper towels & 19.05 & 17.65 & 89.29 & 100 & - & - \\
\hline \multicolumn{2}{|l|}{ Total units } & 524 & 348 & 204 & 70 & 20 & 4 \\
\hline \multicolumn{2}{|l|}{ Success units } & 176 & 144 & 134 & 50 & 16 & 4 \\
\hline \multicolumn{2}{|l|}{ Success rates $(\%)$} & 33.59 & 41.38 & 65.69 & 71.43 & 80 & 100 \\
\hline \multicolumn{2}{|l|}{ Successful criteria } & 2 & 1 & 3 & 5 & 2 & 1 \\
\hline \multicolumn{2}{|c|}{ Criteria with $>70 \%$ success rate } & 0 & 3 & 4 & 2 & 0 & 0 \\
\hline
\end{tabular}

14(hands are dried using paper towels). In Attempt 4, as mentioned before, 5 successful criteria were seen-2 (nails are short and without nail extensions and varnish), 4, 12, 13 and 14 , and $>70 \%$ success rate was seen in 2 criteria -5 (hands are wet under continuously running water) and 6 (warm water is used to wash hands). However, as hand washing is an extremely important practice in the surgical setting, a $100 \%$ efficiency rate was targeted in the project, thus making Attempt 4 (4 successful criteria) more significant than Attempt 3 (3 successful criteria). In Attempt 5, 100\% success rates were documented in criteria 5 and 6 . Worst adherence was noticed in criteria 12 , that is, proper hand washing technique, and it was also the most resilient against change being the only aspect of hand washing requiring six attempts and 5 interventions for $100 \%$ success.

\section{Discussion}

Hand washing is defined by the WHO guidelines on Hand Hygiene in Health Care as washing hands with plain or antimicrobial soap and water [1]. With the advent of hand washing in a healthcare setup in early nineteenth century, it has remained as one of the most valuable practices in prevention of nosocomial infections. The practice of hand cleansing with chloride compounds as documented by Ignaz Semmelweis in an obstetrics clinic in 1846-1847 has evolved over decades to give rise to the concept of 'hand hygiene' used widely in current clinical setting. 'Hand hygiene' is a general term referring to any action of hand cleansing which includes various components of hand washing, hand rubbing and hand care [1]. In the history of hand hygiene, surgical hand antisepsis deserves special mention as invasive procedures quite understandably are associated with increased risk of transmission of infective organisms from surgeons to patients and results in surgical site infections and sepsis [11]. Joseph Lister had documented the effect of hand disinfection with carbolic acid on reduction of wound infections from 1827 to 1912 [12]. Surgical hand washing with antiseptic hand wash was found to be broad spectrum but slow acting and less efficacious, whereas antiseptic hand rubs (alcohol based) were broad spectrum and fast acting but were with less persistent activity [1]. US guidelines summed that agents used for surgical hand scrubs should be a non-irritant antimicrobial agent that significantly reduces microorganisms on intact skin, have broadspectrum activity, and be fast-acting and persistent $[13,14]$.

The data collection template given in the study published on October 2018 named 'Hand Hygiene - Quality Improvement Toolkit for Infection Prevention and Control in General Practice' formulated by Primary 
Care Quality and Information Service under the National Public Health Service for Wales has been used as a reference standard for this study [9]. In the abovementioned study, an effective hand washing technique has been shown to involve three stages - hand preparation, washing and rinsing and drying. A total of 14 criteria for optimum hand washing were suggested, and these have been monitored in the current audit cycle. The current study evaluated 42 surgical internees for compliance to hand washing guidelines shown in Table 1. It was seen that none of the participants were able to perform ideal hand washing in the first attempt, and data collected in Attempt 1 prior to any interventions showed a compliance rate of only $33.59 \%$ across the 14 criteria. This is well below the compliance rates in the study conducted by Basurrah et al. $(69.2 \%$ in internees) but higher than that found in the studies conducted by Salemi et al. (19\%) and Chittaro et al. $(19.3 \%)$ [15-17].

Weber et al. in their study reported video-based instructions as being superior to conventional teaching methods for surgical hand disinfection [18]. In our study, the video demonstration done as the first intervention resulted in 100\% compliance in the category of liquid soap on hand - an increase by $26.19 \%$ from the previous attempt. It also resulted in more than $70 \%$ compliance rate in four other categories. However, in our study, it was seen that demonstration of proper hand washing technique by a consultant (Intervention 3) to the individual participants and addressing their individual drawbacks was the most effective intervention in achieving better compliance to ideal protocols. It led to $100 \%$ compliance rates in five categories of hand washing, with a maximum increase of $33.3 \%$ in the category involving clipping of nails prior to surgical hand washing. The importance of individual guidance in surgical hand washing noted in our study is in agreement to the findings of Salemi et al., in which $70 \%$ of participants were able to recall the personal presentations [16]. In the stage of hand preparation, nail varnish or artificial nails are regarded as major risk factors for transmitting infections by altering the growth of normal skin flora and have been thoroughly discouraged in a clinical setting [19-21]. In addition, surgical teams should avoid any form of hand jewellery (except nonstoned wedding rings) or watches to maintain optimum hand hygiene $[9,20,22,23]$. In the current study, it was noticed that it took four attempts for all the participants to achieve optimum nail hygiene during surgical hand washing. The 14 participants who initially were wearing some form of hand jewellery during hand washing took three attempts to comply with the guidelines. Any cuts or portions of damaged skin are prone to harbour increased number of microorganisms leading to an increased risk of cross infection between the care giver and the patient. Thus water-proof dressing of cuts before surgical hand washing is essential [24]. However, it took four attempts for the 6 participants of this study having cuts on their hands to achieve $100 \%$ compliance rate in this section.

In the stage of hand washing, the hands should be first made wet with tepid running water and only then followed by the application of soap/hand wash, which should come in contact with all the surfaces of the hand and rubbed to form adequate lather [9]. In this study, it was found that following this correct sequence of activities during hand washing was difficult for the participants with uniform application of liquid soap on hand, formation of lather and wetting hands before application of soap took respectively two, three and five attempts to master. However, as the medical institution has a universal supply of warm water to all the operation theatres and wall charts showing proper hand washing technique are hung near the wash basin outside the elective general surgical operation theatres, a $100 \%$ compliance in these two fields have been noted in the first attempt. Even though the use of warm water has been mentioned as a criterion under optimum hand washing in the template used in this study, water temperature has not been found to be a critical factor in removal of microorganisms from the hand during hand washing according to the WHO guidelines published in 2009 [1]. In contrast, in a study comparing water of various temperatures $\left(4{ }^{\circ} \mathrm{C}\right.$, $20^{\circ} \mathrm{C}$ and $40^{\circ} \mathrm{C}$ ) used in hand washing showed a significant level of skin irritation associated with higher temperatures of water as water of higher temperatures are found to remove protective fatty acids from the skin of the hand [25]. In our study, the ten steps of optimum hand washing required the most number of attempts (six) to be completely corrected. As audit cycles on hand washing including these criteria have not been discovered in the literature search, correlation of these findings with worldwide prevalence could not be performed.

Proper method of hand drying after hand washing is equally important as in this stage, there might be recontamination of hands with infective agents [26]. One study compared four methods of hand drying: cloth towels from a roller; paper towels left on a sink; warm air dryer; and letting hands dry by evaporation; no significant difference in the efficacy of the methods was reported [27]. However, articles contradicting this finding are available, and further research in this field needs to be performed before formulation of a universal guideline can be done [28, 29]. If any type of towel is used for hand drying, it must be discarded without touching the disposal bins to prevent recontamination [9]. Sterile cloth towels are used in our institution, and both turning off the tap and hand drying with towels took four attempts in our audit to achieve $100 \%$ efficacy. Recognizing this aspect of hand hygiene is predicted to improve practices related to hand hygiene in health sectors [26]. 
In light of the current global health crisis, proper hand hygiene in all healthcare providers assumes an even greater significance. With a case fatality rate of $2.3 \%$ reported by the Chinese CDC, COVID-19 represents one of the greatest public health emergencies in recent times [30]. It is known to spread via droplet transmission and by fomites, thereby leaving healthcare providers at great risk of contracting the virus themselves, as well as spreading it further amongst their patients and the community at large [6]. Proper hand washing practices thus can be a powerful weapon to help control this pandemic not just in surgical specialities, but for other medical disciplines as well.

Hand washing is a well-documented method to prevent surgical site infections, with baseline data showing suboptimal compliance in our institution. The results of this audit are a representation of the efficacy of the use of instructional videos and personal supervision as tools for attaining $100 \%$ compliance in the field of surgical hand washing. This study model can be expanded to achieve complete departmental coverage and can also act as a template for similar studies in the future. However, an objective microbiological distribution analysis along with appropriate follow-up is needed for assessing the long-term benefit arising from the programme. The use of CCTV cameras can also be made to limit any observation bias.

Authors' Contributions Ramanuj Mukherjee: Conceptualization and study design

Pritha Roy: Manuscript writing and review of literature

Madhav Parik: Statistical analysis, manuscript writing and correspondence

\section{Compliance with Ethical Standards}

Conflict of Interest The authors declare that they have no conflicts of interest.

\section{References}

1. World Health Organization (WHO). WHO guidelines on hand hygiene in health care 2009. Available from https://www.who.int/ gpsc/5may/tools/9789241597906/en/. Accessed 22 Jun 2020

2. Tanner J (2008) Surgical hand antisepsis: the evidence. J Perioper Pract. 18(8):330-334 339

3. Pirie S (2010) Hand washing and surgical hand antisepsis. J Perioper Pract 20(5):169-172

4. Allegranzi B, Pittet D (2007) Healthcare-associated infection in developing countries: simple solutions to meet complex challenges. Infect Control Hosp Epidemiol 28:1323-1327

5. Cascella M, Rajnik M, Cuomo A, et al Features, evaluation and treatment coronavirus (COVID-19) [Updated 2020 Apr 6]. In: StatPearls [Internet]. Treasure Island (FL): StatPearls Publishing; 2020 Jan-. Available from: https://www.ncbi.nlm.nih.gov/books/ NBK554776/. Accessed 22 Jun 2020

6. National Patient Safety Agency (NPSA) Patient safety alert Second Edition 2 September 2008: The WHO five moments for hand hygiene. http://www.npsa.nhs.uk/cleanyourhands/the-campaign/ latest-news/. Accessed 22 Jun 2020

7. Sánchez-Payá J, Galicia-García MD, Gracia-Rodríguez RM, García-González C, Fuster-Pérez M, López-Fresneña N, Avendaño-Corcoles F, González-Torga A (2007) Compliance with hand hygiene guidelines and determinants of compliance. Enferm Infecc Microbiol Clin 25(6):369-375

8. Ezzat A, Safdar MM, Ahmed I (2014) Are we following the WHO recommendations for surgical scrubbing? Scott Med J 59(4):214 219

9. HAND HYGIENE quality improvement toolkit for Infection Prevention \& Control in general practice. National Public Health Service for Wales; 2008

10. WHO (2015) WHO: how to handwash? With soap and water [video] Available at: https://www.youtube.com/watch?v= 3PmVJQUCm4E\&t=7s. Accessed 20 May 2020

11. Centers for Disease Control and Prevention.Guideline for Hand Hygiene in Health-Care Settings: Recommendations of the Healthcare Infection Control Practices Advisory Committee and the HICPAC/SHEA/APIC/IDSA Hand Hygiene Task Force. MMWR 2002;51(No. RR-16):[17]

12. Maki DG (1976) Lister revisited: surgical antisepsis and asepsis. N Engl J Med 294:1286-1287

13. Vincent JL, Bihari DJ, Suter PM, Bruining HA, White J, NicolasChanoin MH, Wolff M, Spencer RC, Hemmer M (1995) The prevalence of nosocomial infection in intensive care units in Europe. Results of the European prevalence of infection in intensive care (EPIC) study. EPIC International Advisory Committee. JAMA 274:639-644

14. Weber DJ, Rutala WA, Blanchet CN, Jordan M, Gergen MF (1999) Faucet aerators: a source of patient colonization with Stenotrophomonas maltophilia. Am J Infect Control 27:59-63

15. Basurrah MM, Madani TA (2006) Hand washing and gloving practice among health care workers in medical and surgical wards in a tertiary care centre in Riyadh, Saudi Arabia. Scand J Infect Dis 38(8):620-624

16. Salemi C, Canola MT, Eck EK (2002) Hand washing and physicians: how to get them together.osp. Epidemiol 23(1):32-35

17. Chittaro M, Coiz F, Faruzzo A, Fiappo E, Palese A, Viale P, Brusaferro S (2006) Compliance with hand washing in health care settings. Ann Ig 18(2):109-115

18. Weber U, Constantinescu MA, Woermann U, Schmitz F, Schnabel K (2016) Video-based instructions for surgical hand disinfection as a replacement for conventional tuition? A randomised, blind comparative study. GMS J Med Educ 33(4):Doc57

19. McNeil SA et al (2001) Effect of hand cleansing with antimicrobial soap or alcohol-based gel on microbial colonization of artificial fingernails worn by health care workers. Clin Infect Dis 32:367372

20. Guidelines on hand hygiene in health care (2006) Current issues and forthcoming events. J Adv Nurs 53:613-614

21. Hedderwick SA, McNeil SA, Kauffman CA (2000) Pathogenic organisms associated with artificial fingernails worn by healthcare workers. Infect Control Hosp Epidemiol 21:505-509

22. Boyce JM, Pittet D (2002) Guideline for hand hygiene in healthcare settings. Recommendations of the healthcare infection control practices advisory committee and the HICPAC/ SHEA/APIC/IDSA hand hygiene task force. Morb Mortal Wkly Rep 51:1-45

23. Rotter ML (2004) European norms in hand hygiene. J Hosp Infect 56(Suppl. 2):S6-S9

24. National Institute for Clinical Excellence (2003) Infection control prevention of healthcare associated infection in primary and community care. ht t p :// w w w.org.uk/pdf/ CG2fullguidelineinfectioncontrol.pdf. Accessed 20 Jun 2020 
25. Berardesca E, Vignoli GP, Distante F, Brizzi P, Rabbiosi G (1995) Effects of water temperature on surfactant-induced skin irritation. Contact Dermatitis 32:83-87

26. Patrick DR, Findon G, Miller TE (1997) Residual moisture determines the level of touch-contact-associated bacterial transfer following hand washing. Epidemiol Infect 119:319-325

27. Gustafson DR, Vetter EA, Larson DR, Ilstrup DM, Maker MD, Thompson RL, Cockerill FR 3rd (2000) Effects of 4 hand-drying methods for removing bacteria from washed hands: a randomized trial. Mayo Clin Proc 75:705-708

28. Yamamoto Y, Ugai K, Takahashi Y (2005) Efficiency of hand drying for removing bacteria from washed hands: comparison of paper towel drying with warm air drying. Infect Control Hosp Epidemiol 26:316-320

29. Ngeow YF, Ong HW, Tan P (1989) Dispersal of bacteria by an electric air hand dryer. Malays J Pathol 11:53-56

30. The Novel Coronavirus Pneumonia Emergency Response Epidemiology Team (2020) The epidemiological characteristics of an outbreak of 2019 novel coronavirus disease (COVID-19) - China, 2020. China CDC Weekly 2:1-10

Publisher's Note Springer Nature remains neutral with regard to jurisdictional claims in published maps and institutional affiliations. 